\title{
Development of Gas Cluster Ion Beam Surface Treatments for Reducing Field Emission and Breakdown in RF cavities
}

\author{
D.R. Swenson ${ }^{1}$, A.T. $\mathrm{Wu}^{2}$, E. Degenkolb ${ }^{1}$ and. Z Insepov ${ }^{3}$ \\ ${ }^{1}$ Beam Physics, Epion Corporation, 37 Manning Road, Billerica MA 01821, USA \\ ${ }^{2}$ Jefferson National Laboratory, 12000 Jefferson Ave., Newport News VA 23606, USA \\ ${ }^{3}$ Argonne National Laboratory, 9700 South Cass Ave., Argonne IL 60439, USA
}

\begin{abstract}
Sub-micron-scale surface roughness and contamination cause field emission that can lead to high voltage breakdown of electrodes, and these are limiting factors in the development of high gradient RF technology. We are studying various Gas Cluster Ion Beam (GCIB) treatments to smooth, clean, etch and/or chemically alter electrode surfaces to allow higher fields and accelerating gradients, and to reduce the time and cost of conditioning high voltage electrodes. For this paper, we have processed Nb, Stainless Steel, and Ti electrode materials using beams of $\mathrm{Ar}, \mathrm{O}_{2}$, or $\mathrm{NF}_{3}+\mathrm{O}_{2}$ clusters with accelerating potentials up to $35 \mathrm{kV}$. Using a Scanning Field Emission Microscope (SFEM), we have repeatedly seen a dramatic reduction in the number of field emission sites on $\mathrm{Nb}$ coupons treated with GCIB. Smoothing effects on Stainless steel and Ti substrates have been evaluated using AFM imaging and show that 200-nm wide polishing scratch marks are greatly attenuated. A 150-mm diameter GCIB treated stainless steel electrode has now shown virtually no DC field emission current at gradients over 20 $\mathrm{MV} / \mathrm{m}$.
\end{abstract}

Keywords: cluster-surface interactions, gas cluster ion beam, RF breakdown, superconducting RF cavity, field emission

PACS: 36:40.-c, 29.17.+w, 68.37.Vj, 68.90.+g, 68.49.Fg

\section{INTRODUCTION}

Most of the recent increases in Superconducting RF (SRF) cavity acceleration gradients are the result of better techniques for preparing the cavity surfaces. The physical and chemical characteristics of the thin layer defined by the skin-depth of the microwaves $(\approx 50 \mathrm{~nm})$ are critical to achieving higher gradients. Techniques such as $\mathrm{BCP}$, EP, vacuum baking, rinsing with high-pressure high-purity water, cavity assembly in clean rooms, high pulsed power conditioning, and the use of single or large crystal $\mathrm{Nb}$ are used to reduce roughness, defects, particles and contamination that cause field emission, quenching, "high field Q-slope", secondary emission, and breakdown [1-3]. Further advances are needed to meet the goal of $35 \mathrm{MV} / \mathrm{m}$ for the proposed International Linear Collider (ILC) that will probe for new physics using $\mathrm{TeV}$ collisions of electron and positron beams. We are investigating electrode preparation using Gas Cluster Ion Beams (GCIB) a new nano-technology for smoothing, cleaning, or chemically altering surfaces that is capable of achieving atomic level smoothness on planar and non-planar surfaces [4]. With GCIB

\author{
CP877, Advanced Accelerator Concepts: $12^{\text {th }}$ Workshop, \\ edited by $\mathrm{M}$. Conde and C. Eyberger \\ (C) 2006 American Institute of Physics 978-0-7354-0378-9/06/\$23.00
}


processing the surface is bombarded with an energetic beam of nano-sized cluster ions. The clusters are formed as a high-pressure gas ( $\approx 15 \mathrm{bar})$ is emitted through a small orifice into a nozzle located in a region of high vacuum $\left(\approx 3 \times 10^{-6} \mathrm{bar}\right)$. The gas cools during the adiabatic expansion and condenses and coagulates into a jet of clusters. The jet passes through a differential pumping aperture; the clusters are ionized by electron impacts, and then electrostatically accelerated (using potentials as high as $35 \mathrm{kV}$ in these experiments). A dipole magnet deflects monomers and dimers out of the beam. Typically the treated substrate is mechanically scanned in the beam to assure uniform irradiation of the sample $[5,6]$. Average parameters for the clusters in an Ar GCIB beam accelerated with $30 \mathrm{kV}$ are mass $=10,000$ atoms, charge state $=+3.2$, velocity $=6.7 \mathrm{~km} / \mathrm{s}$ with a beam current of $>200 \mu \mathrm{A}[7,8]$. GCIB treatments are used industrially in the production of thin-film optical coatings, fixed disk memory technology, EUV lithography masks, and for the manufacture of semiconductor devices [9]. Previous papers have reported the results of GCIB treatments of OFE-Cu, stainless steel, $\mathrm{Ti}$ and $\mathrm{Nb}$ electrode surfaces [10-12]. This paper contains additional data for stainless steel electrodes and reports the direct measurements of the suppression of field emission by GCIB treatments of Nb SRF cavity material.

\section{Stainless Steel Electrode Material}

Samples of highly polished stainless steel electrode material used for extraction electrodes of high-field photoelectron guns [13] were provided by Cornell Wilson Laboratory. These samples were carefully hand-polished using diamond paste to an average roughness of less than $1 \mu \mathrm{m}$ and had a mirror like appearance. However these surfaces are not smooth at nanometer and atomic dimensions as can be seen in Fig. 1, a typical AFM image of these surfaces. The surface shows isolated asperities and sharp-edged, $\approx 200$-nm-wide scratch marks produced by the polishing compound. The samples were treated with a combination of high and low energy Ar GCIB followed by high and low energy $\mathrm{O}_{2}$ GCIB. Figure 2 is an AFM image of the same surface after treatment showing that GCIB effectively removes the asperities and scratch marks.

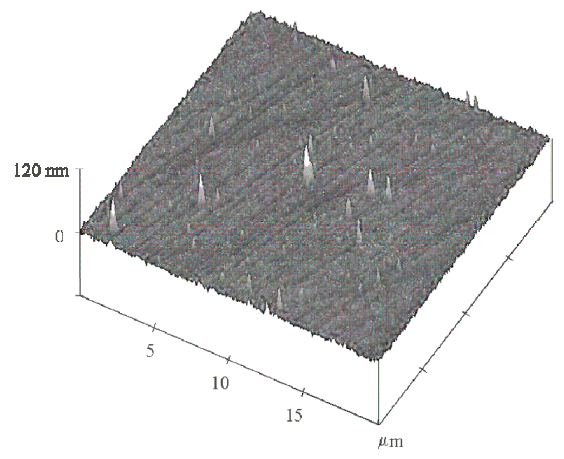

FIGURE 1. AFM image $(20 \times 20 \mu \mathrm{m})$ of highly polished stainless steel electrode material before GCIB treatment showing asperities and scratch marks from polishing. The vertical scale is $120 \mathrm{~nm} /$ division. 


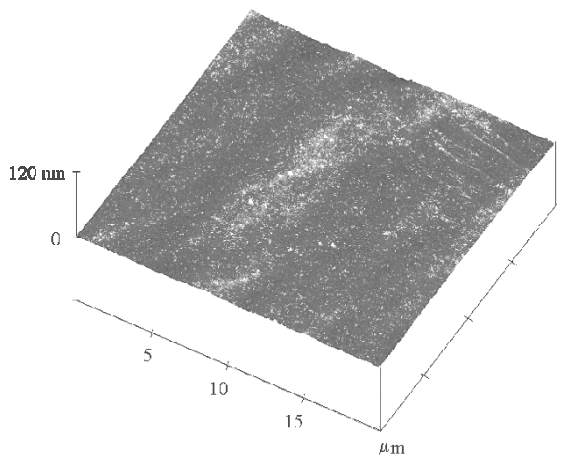

FIGURE 2. AFM image $(20 \times 20 \mu \mathrm{m})$ of highly polished Stainless steel electrode material after GCIB treatment. The vertical scale is $120 \mathrm{~nm} /$ division.

Figure 3 shows Power Spectral Density (PSD) analysis of these two images and other AFM measurements of these surfaces. The PSD's are obtained from the Fourier transforms of the pixilated height maps made by the AFM, and they show the relative effectiveness of GCIB processing for different size scales of roughness. GCIB processing is particularly effective for roughness with wavelengths up to $600 \mathrm{~nm}$, and continues to be effective up to roughness of $2 \mu \mathrm{m}$ wavelength in these measurements. This is the scale of roughness that is expected to contribute to field emission, and this is direct evidence that GCIB is effective at smoothing these features that could not be smoothed even using the best of previous mechanical polishing techniques for non planar electrodes. The effective size scale for GCIB polishing is determined by: the properties of the surface, the average size of craters made by individual cluster impacts (typically in the range of 10-20 nm for high energy Ar clusters [14-18]), and by the applied GCIB dose. We have seen very similar results when treating $\mathrm{Ti}$ surfaces with similar initial polish using Ar GCIB.

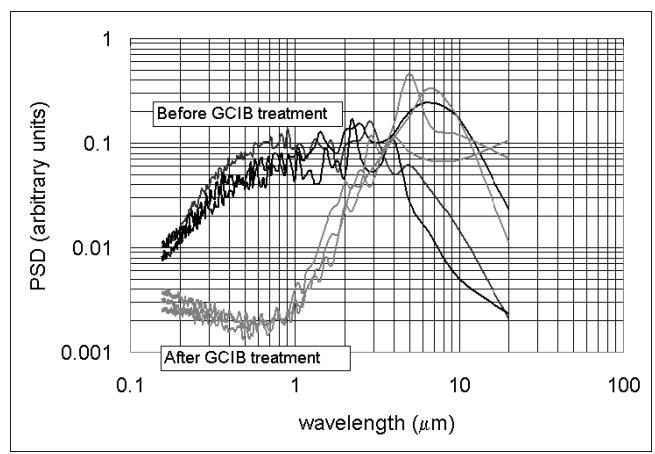

FIGURE 3. Six plots of 2-dimensional isotropic PSD analysis of 20x20 $\mu \mathrm{m}$ AFM images showing the affect of GCIB processing on highly polished stainless steel electrode material. The three black lines are before processing and the three gray lines are after processing. 


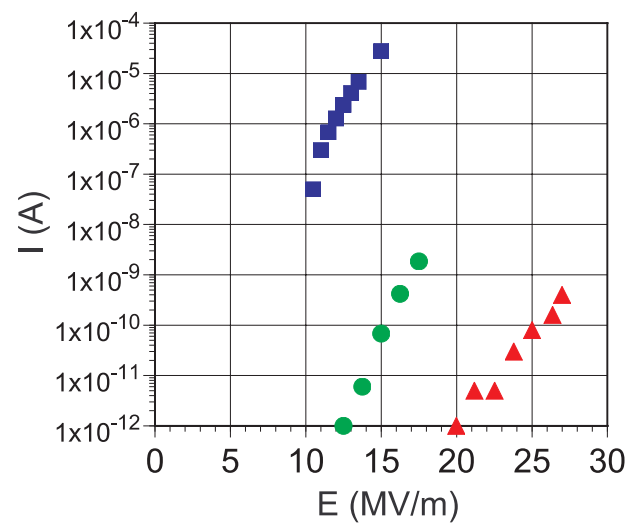

FIGURE 4 Field emission current as a function of applied gradient for 150-mm-diameter stainless steel electrodes: a typical untreated sample (squares), first measurement of a GCIB treated sample (circles), re-measurement of GCIB treated sample (triangles) after hi-potting [19].

Previously we reported measurements showing a factor of $10^{6}$ reduction of field emission resulting from GCIB treatment for a $150-\mathrm{mm}$ diameter stainless-steel electrode that was polished similar to that of Figs 1-3 [12]. The field emission was measured at the Jefferson Laboratory Large Area Electrode Test Chamber. This same electrode was remeasured several months later on an identical test stand at Cornell Wilson Laboratory. Whereas the first measurement was swiftly made because of time constraints, this second measurement allowed more time for high voltage conditioning of the electrode (hi-potting). Not only did the electrode maintain its original performance but, with hi-potting, a gradient of over $20 \mathrm{MV} / \mathrm{m}$ was reached with no measurable field emission, as plotted in Fig. 4 [19]. It should also be noted that the processing of this electrode included treatments with $\mathrm{O}_{2}$ clusters, that increased the thickness of the surface oxide layer from $1.5 \mathrm{~nm}$ to $>10 \mathrm{~nm}$ [12]. The treated surface was also measured to be twice as hard as the untreated surface [12]. The relative contributions of smoothing, oxide thickness, and surface hardening to the reduction in the field emission is receiving further study.

\section{Nb Material for SRF Cavities}

Samples of $\mathrm{Nb}$ SRF cavity material were supplied by Jefferson laboratory, and were GCIB treated at Epion, and then returned to Jefferson laboratory for study. These 25-mm diameter coupon samples were prepared using Buffered Chemical Polishing (BCP) that is typically used for SRF cavities [1-3]. Field emission from the samples was studied using the Scanning Field Emission Microscope (SFEM) located at the Surface Science Laboratory [20]. The SFEM automatically maps the locations of field emitters on the surface of a sample by raster scanning a biased micro-tip over the surface. At each point on the surface the threshold for field emission is determined by ramping the tip voltage until a specified current level is reached. The system also 


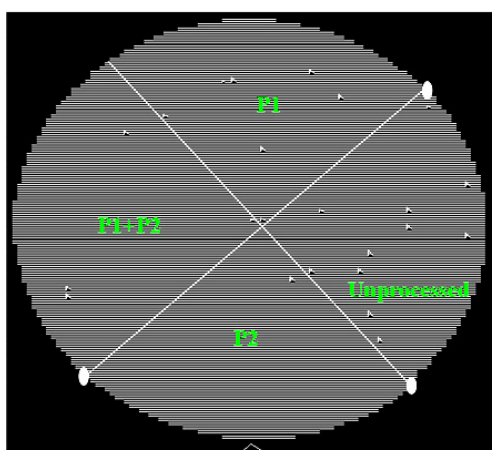

FIGURE 5 SFEM Plot of field emitters on surface of a 25-mm diameter coupon sample of BCPpolished $\mathrm{Nb}$ SRF cavity material. The sample was masked into equal quadrants for treatment with $\mathrm{Ar}$ (P1) and/or $\mathrm{O}_{2}$ (P2) GCIB beams or not treated as designated on the plot.

allows the samples to be transferred under high vacuum into an SEM for imaging, and for study of elemental composition using EDX. The samples are indexed so that field emitters found by the SFEM scan can be relocated and studied using the SEM and EDX. The samples were handled at Jefferson laboratory and at Epion Corporation using particle-free gloveboxes to minimize contamination. A $25-\mu \mathrm{m}$ thick stainless steel mask located approximately $2 \mathrm{~mm}$ above the surface was used to shield a portion of the sample from the GCIB processing. Figure 5 is the first SFEM map of a GCIB treated $\mathrm{Nb}$ sample. The sample was masked into quadrants that received either: no processing, Ar processing, $\mathrm{O}_{2}$ processing, or a combination of $\mathrm{Ar}$ then $\mathrm{O}_{2}$ processing. All three treated quadrants showed fewer field emitters than the unprocessed quadrant. The untreated quadrant had 11 field emitters while the $\mathrm{O}_{2}$ treated quadrant had only one field emitter. Comparing these results to a binomial distribution showed less than a 1 in 70 chance that this is a random distribution. The $\mathrm{O}_{2}$ treatment was retested and again, Fig. 6, there was a dramatic reduction in the number of field emitters, confirming the previous result. On the second sample a total of 23 field emitters were found on the untreated half of the sample and only 3 on the treated half. Preliminary SEM and SIMS studies show evidence that particles on the surface may be disrupted

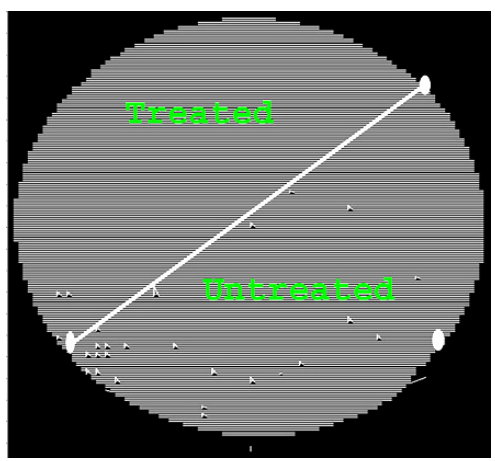

FIGURE 6 SFEM plot of field emitters on surface of a 25-mm diameter coupon sample of BCPpolished $\mathrm{Nb}$ SRF cavity material. Half of the sample was treated with $\mathrm{O}_{2} \mathrm{GCIB}$ beams. 
by the $\mathrm{O}_{2}$ treatment and that the oxygen stoichiometry of the oxide may be increased. It is to be expected that a hypervelocity collision of an oxygen cluster containing several thousand molecules will cause a powerful exothermic chemical reaction on metallic, hydrocarbon and other reactive surfaces in addition to the kinetic collision energy. These "nano-explosions" should be disruptive to particulates because they are structurally weak and thermally isolated. The effect of these reactions on the $\mathrm{Nb}$ substrate is discussed in another paper [21].

An earlier study concluded that $\mathrm{NF}_{3}+\mathrm{O}_{2}$ clusters can significantly etch $\mathrm{Nb}$ and blunt the angles of the grains that protrude from the surface [12]. The conclusion was based on multiple AFM measurements of the grain edges and SEM images. Figure 7 is a SFEM map of a sample treated with $\mathrm{NF}_{3}+\mathrm{O}_{2}$ clusters. The treated half also shows many fewer field emitters than the untreated half (the results are even more statistically significant than in the case of $\mathrm{O}_{2}$; the result is 4 standard deviations from the mean for the binomial distribution). The sample was also studied using a scanning two-dimensional profilometer but this instrument did not show significant change in the average roughness measured over a $1,500 \times 1,500 \mu \mathrm{m}$ scan area. That the range of heights did not decrease disagrees with our previous findings of possible grain removal [12]. The resolution of this measurement was insufficient to test blunting of the angles of the exposed grains.

For GCIB treatments to be practical for processing of SRF cavities for the proposed ILC, that will have approximately 16,000 1-meter-long Nb SRF acceleration cavities, requires reasonable processing times. We have measured the etch rate of $\mathrm{Nb}$ using a quartz crystal microbalance (Inficon XTM/2) for various GCIB beams produced by our test stand. The test stand has less than half the etch rate of the standard Epion Ultrasmoother ${ }^{\mathrm{TM}}$ product, and the processing has not been optimized, so the results that are plotted in Fig. 8 are very conservative estimates of processing rates. The beam current for Ar accelerated with $30 \mathrm{kV}$ was $93 \mu \mathrm{A}$ and the sputter yield was $8.6 \mathrm{Nb}$ atoms/e. The low sputter yield [22] is most likely a result of the higher beamline pressure of the test stand. Higher pressure causes more cluster-gas collisions with the residual gas and these collisions abrade the clusters [7, 8, 24]. For smoothing

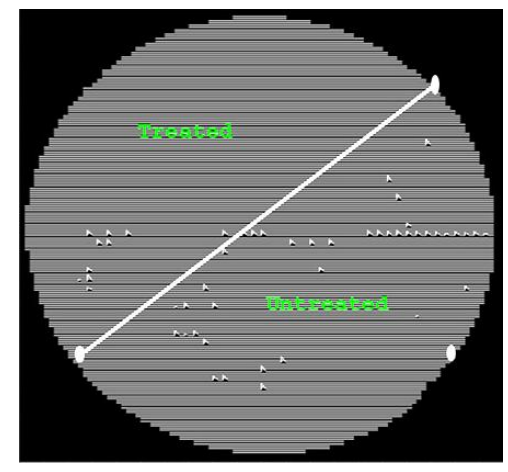

FIGURE 7 SFEM plot of field emitters on the surface of a $25-\mathrm{mm}$ diameter coupon sample of BCPpolished $\mathrm{Nb} \mathrm{SRF}$ cavity material. Half of the sample was treated with $\mathrm{NF}_{3}+\mathrm{O}_{2} \mathrm{GCIB}$ beams. 


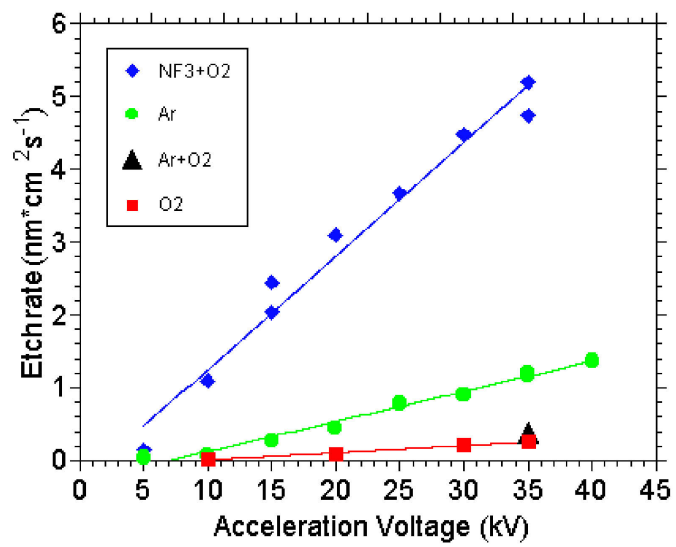

FIGURE 8 The etch rate $\left(\mathrm{nm}^{*} \mathrm{~cm}^{2} * \mathrm{~s}^{-1}\right)$ of $\mathrm{Nb}$ by $\mathrm{NF}_{3}+\mathrm{O}_{2}, \mathrm{Ar}, \mathrm{Ar}+\mathrm{O}_{2}$ [22], and $\mathrm{O}_{2}$ GCIB beams produced on a test stand as a function of acceleration voltage.

cavities for the ILC, a quick calculation shows that, assuming an etch rate of $5 \mathrm{~nm} * \mathrm{~cm}^{2} / \mathrm{s}$, less than 9 hours is required to remove $50 \mathrm{~nm}$ (approximately the skin depth of the RF) from the surface of a cylindrical cavity that is 1-meter long and $10-\mathrm{cm}$ in diameter. Thus the entire active volume of a cavity could be removed in a few hours, a time comparable to that required for many of the processing steps now in use. Conductance calculations show that acceptable beamline pressures can be maintained inside the cavity during processing. With optimization of the process and better equipment the process times can be substantially reduced.

\section{CONCLUSIONS}

It is evident that GCIB processing is particularly effective at smoothing roughness of size scales less than $1 \mu \mathrm{m}$, and we have previously shown accelerated etching of isolated asperities $300 \mathrm{~nm}$ diameter and $35 \mathrm{~nm}$ high [8]. Thus GCIB processing is uniquely capable of smoothing roughness of the scale of the field emitters that have been identified on electrodes [25-27]. There is also strong evidence that GCIB can dramatically suppress field emitters on Nb SRF cavity surfaces. With this compelling evidence, Epion Corporation has begun the design of equipment for in-situ treatment of $\mathrm{Nb}$ SRF cavities. Finally, it should be noted that while achieving a $20 \mathrm{MV} / \mathrm{m}$ gradient without appreciable field emission is exceptional, a quick calculation using Fowler-Nordheim theory for an ideal electrode, assuming a workfunction of $4.5 \mathrm{eV}$, an emitter area of $115 \mathrm{~cm}^{2}$ and a local field intensification factor $\beta$ of 1 , yields a field emission threshold of over $1 \mathrm{GV} / \mathrm{m}$. Thus we are far from the ideal electrode and large improvements can be expected. 


\section{ACKNOWLEDGMENTS}

We thank Dr. C.K. Sinclair and co-workers for helpful discussions and for providing the samples and measurements of field emission for the stainless steel electrodes. This work was supported in part by DE-FG02-04ER83944.

\section{REFERENCES}

1. H. Padamsee, Supercond. Sci. Technol. 14, R28 (2001).

2. P. Kneisel, NASA Scientific and Technical Aerospace Reports, 42(18), 2004, Report No.: DE2004822207.

3. D. Proch, Rep. Prog. Phys. 61, 431 (1998).

4. I. Yamada, J. Matsuo, N. Toyoda, A. Kirkpatrick, Mater. Sci. Eng. R 34, 231 (2001).

5. M.E. Mack, R. Becker, M. Gwinn, D.R. Swenson, R.P. Torti, R. Roby, Proceedings of the 14th International Conference on Ion Implantation Technology, Taos, NM 2002, IEEE, 89 (2003).

6. M. E. Mack, Nucl. Instrum. Methods B 237, 235 (2005).

7. D.R. Swenson, Nucl. Instrum. Methods B 222, 61 (2004).

8. D.R. Swenson, Nucl. Instrum. Methods B 241, 599 (2005).

9. R. MacCrimmon, J. Hautala, M. Gwinn, S. Sherman, Nucl. Instr. Meth. B 242, 427 (2006).

10. D.R. Swenson, E. Degenkolb, Z. Insepov, L. Laurent, G. Scheitrum, Nucl. Instrum. Methods B 241, 641 (2005).

11. Z. Insepov, A. Hassanein, D. R. Swenson, and M. Terasawa, Nucl. Instrum. Methods B 241, 496 (2005).

12. D.R. Swenson, E. Degenkolb, and Z. Insepov, Physica C 441, 75 (2006).

13. C.K. Sinclair, Proceedings of the 2003 Particle Accelerator Conference, p. 78, (2003).

14. Z. Insepov, L.P. Allen, C. Santeufemio, K.S. Jones, I. Yamada, Nucl. Instrum. Methods B 206, 846 (2003).

15. L.P. Allen, Z. Insepov, D. B. Fenner, C. Santeufemio, W. Brooks, K. S. Jones and I. Yamada, J. Appl. Phys. 92, 3671 (2002).

16. O. Rattunde M. Moseler, A. Häfele, J. Kraft, D. Rieser, and H. Haberland, J. Appl. Phys. 90, 3225 (2001).

17. N. Toyoda, N. Hagiwara, J. Matsuo, I. Yamada, Nucl. Instrum. Methods B 151, 980 (2000).

18. W.K. Chu, Y.P. Li, J.R. Liu, J.Z. Wu, S.N. Tidrow, J. Matsuo and I. Yamada, Appl. Phys. Lett. 72, 246 (1998).

19. C.K. Sinclair, private communication.

20. A.T. Wu, Proceedings of the 11th Workshop on RF Superconductivity, Germany, (2003) ThP13.

21. Z. Insepov, A. Hassanein, J. Norem, and D.R. Swenson, these proceedings.

22. J. Matsuo, N. Toyoda, M. Akizuki, and I. Yamada, Nucl. Instrum. Methods B 121, 459 (1997).

23. M. Saito, N. Hagiwara, N. Toyoda, J. Matsuo and I. Yamada, Proceedings 1998 International Conference on Ion Implantation Technology, IEEE, p. 1226 (1999).

24. T. Seki and J. Matsuo, Nucl. Instrum. Methods B 241, 604 (2005).

25. R. Latham, High Voltage Vacuum Insulation, Academic Press, London, 1995, p. 27.

26. J. Norem, A. Hassanein, I. Konkashbaev, Proceedings of the 2003 Particle Accelerator Conference, p. 1180 (2003).

27. J. Norem, Z. Insepov, I. Konkashbaev, Nucl. Instrum. Methods A 537, 510 (2005). 